\title{
ON PRIMARY IDEALS IN $C(X)$
}

J. G. HORNE, JR.

1. Introduction. Let $X$ be a topological space. We show that an ideal $P$ in $C(X)$ is a primary ideal if and only if $P$ contains a maximal $O$-ideal. Assuming that $X$ is a completely regular $G_{\delta}$-space, we obtain several characterizations of fixed maximal ideals of $C(X)$ in terms of principal primary ideals. A connection is established between these characterizations and that obtained by Pursell [8].

2. Primary ideals in $C(X) . C(X)$ denotes the ring of real-valued continuous functions on the topological space $X$, under ordinary pointwise addition and multiplication. By a primary ideal we mean an ideal which is contained in at most one maximal ideal. An O-ideal is an ideal $I$ such that for each pair $f_{1}, f_{2} \in I$ there exists $e \in I$, depending on $f_{1}$ and $f_{2}$, such that $f_{i} e=f_{i}$, for $i=1,2[7 ; 4]$. A prime-like ideal of $C(X)$ is an ideal $P$ such that if $(f, k, e)$ is any triple of elements of $C(X)$ which satisfies $f \notin P, f e=f$ and $k e \in P$, then $k \in P$ [4]. If $J \subset C(X)$ is an ideal, we let $L(J)$ denote the set $\{f \in C(X): f e=f$ for some $e \in J\}$. Obviously $L(J) \subset J$. We shall use these additional results from $[4]$ :

(i) $L(J)$ is an $O$-ideal which is a maximal $O$-ideal if $J$ is a primelike ideal.

(ii) If $N$ is an $O$-ideal in $J$ then $N \subset L(J)$, so if $N$ is a maximal $O$ ideal then $N=L(J)$.

(iii) Every prime ideal is a prime-like ideal.

The following lemma is used in the proof of Theorem 1 and incidentally to yield an alternate proof of a result in [2].

Lemma 1. If $M$ is a maximal ideal in $C(X)$ and $f \in M$, then there is $h \in C(X)$ such that $(h f-1) \in L(M)$.

Proof. If $f \notin M$ there exists $k \in C(X)$ and $m \in M$ such that $k f+m$ $=1$. Choose a real number $t_{1}, 0<t_{1}<1$, and let $t_{2}=1-t_{1}$. Set $h_{1}$ $=1 /\left(k f \bigvee t_{1}\right)$ and $h_{2}=1 /\left(m \bigvee t_{2}\right)$. (In general, $\vee$ and $\wedge$ denote the ordinary lattice operations in $C(X))$. A simple calculation shows $\left(h_{1} k f-1\right)\left(h_{2} m\right)=h_{1} k f-1$. Hence, $\left(h_{1} k f-1\right) \in L(M)$ and $h=h_{1} k$ is the desired function.

It was proved in $\left[2\right.$, p. 342] that the ideals $N^{p}$ are primary ideals. In [4, Example 2.14], it was remarked that the ideals $N^{p}$ are exactly

Presented to the Society, August 30, 1957; received by the editors January 20, 1958. 
the maximum $O$-ideals in $C(X)$. Thus the maximal $O$-ideals in $C(X)$ are primary ideals. The above lemma yields a proof of this fact which makes no reference to the Stone-Cech compactification $\beta(X)$ of $X$. For assume that a given maximal $O$-ideal $N$ is contained in distinct maximal ideals $M_{1}$ and $M_{2}$. By (i) and (ii) above, $L\left(M_{1}\right)=N=L\left(M_{2}\right)$. However, since $M_{1}$ and $M_{2}$ are distinct, there exist elements $m_{1} \in M_{1}$ and $m_{2} \in M_{2}$ such that $m_{1}+m_{2}=1$. Now $m_{2} \notin M_{1}$, so, by the lemma, there exists $h$ such that $\left(h m_{2}-1\right) \in L\left(M_{1}\right)$. But certainly $\left(h m_{2}-1\right)$ $\notin L\left(M_{2}\right)$, which is a contradiction. Hence $N$ is a primary ideal. Actually we can say more.

Theorem 1. For an ideal $P \subset C(X)$, the following conditions are equivalent:

(1) $P$ contains a maximal $O$-ideal;

(2) $P$ is a primary ideal;

(3) $P$ is a prime-like ideal.

Proof. The statement $(1) \rightarrow(2)$ is immediate since any ideal which contains a primary ideal is itself a primary ideal. By (i) above, $(3) \rightarrow(1)$.

We next show that $(2) \rightarrow(1)$. Suppose $P$ is a primary ideal. By Zorn's lemma, $C(X)$ contains a maximal $O$-ideal, so if $P=C(X)$ then $P$ contains a maximal $O$-ideal. If $P \neq C(X)$ then $P$ is contained in some maximal ideal $M$. We shall see that $L(M) \subset P+N$ for every maximal $O$-ideal $N$. If $N=L(M)$ then this result is obvious. Suppose $N \neq L(M)$ and choose $f \in L(M)$ arbitrarily. $N$ is contained in a maximal ideal $M^{\prime}$. Since $L\left(M^{\prime}\right)=N$ we have $M^{\prime} \neq M$. Therefore $P \nsubseteq M^{\prime}$, since $P$ is a primary ideal. Choose $g \in P \backslash M^{\prime}$. By Lemma 1 , there is $h \in C(X)$ such that $(h g-1) \in L\left(M^{\prime}\right)=N$. Thus $f g h-f \in N$ and $f \in N+f g h \subset N+P$. Hence, $L(M) \subset P+N$. Now

$$
P=\cap\{P+N: N \text { is a maximal } O \text {-ideal }\}
$$

[4, Theorems 4.25 and 5.3. and Remark 4.5 (ii)], so $P$ contains the maximal $O$-ideal $L(M)$ and $(2) \rightarrow(1)$.

Finally, $(1) \rightarrow(3)$. For assume that $P$ contains a maximal $O$-ideal $N$. If $P=C(X)$ then $P$ is a prime-like ideal. If $P \neq C(X)$ then $P$ is contained in a maximal ideal $M$. Now $N=L(P)$ by (ii), and $N=L(M)$ since $N \subset P \subset M$. Suppose $f, k$ and $e$ are elements of $C(X)$ such that $f \notin P, f e=f$ and $k e \in P$. Then $e \notin M$, for otherwise $f \in L(M)=L(P) \subset P$. Therefore, by Lemma 1 , there exists a function $h \in C(X)$ such that $(h e-1) \in L(M)$, so $(k h e-k) \in L(M)$. Thus $(k h e-k) \in P$. But khe $=h(k e) \in P$ since $k e \in P$. Hence $k \in P$, so $P$ is a prime-like ideal and the proof of the theorem is complete. 
Note that the statement $(3) \rightarrow(2)$ of the previous theorem generalizes Theorem 3.3 of [2], in virtue of (iii). The following corollary is a generalization of Lemma 3.2 of that paper.

Corollary. If $P$ is any prime-like (=primary) ideal contained in the maximal ideal $M$, then $P \supset L(M)$.

Proof. $P$ contains a maximal $O$-ideal $N$, by the theorem, so $L(P)$ $=N$. On the other hand, $L(M)=N$ since $M \supset N$. Thus $L(P)=L(M)$. But $P$ always contains $L(P)$, so $P \supset L(M)$.

3. Fixed maximal ideals. For a subset $A \subset X$ let $A^{\circ}$ denote the interior of $A$. For $f \in C(X)$, the set $Z(f)=\{x \in X: f(x)=0\}$ is the zero set of $f$; we use $|f|$ to denote the function defined by the identity $|f|(x)=|f(x)|$. The ideal generated by a finite subset $\left\{f_{1}, f_{2}, \cdots, f_{n}\right\}$ of $C(X)$ is denoted by $\left[f_{1}, f_{2}, \cdots, f_{n}\right]$. For $x \in X$, set $M(x)$ $=\{f \in C(X): x \in Z(f)\}$ and $N(x)=\left\{f \in C(X): x \in Z(f)^{\circ}\right\}$. Evidently $N(x)=L(M(x))$ if $X$ is completely regular. For it is immediate that $L(M(x)) \subset N(x)$, while if $x \in Z(f)^{\circ}$, then there is a function $e \in C(X)$ such that $e \in M(x)$ and $e \mid\left(X \backslash Z(f)^{\circ}\right)=1$. For such $e$ we have $f e=f$, so $f \in L(M(x))$. By Theorem 35 of [3], $M(x)$ is a maximal ideal, so $N(x)$ is a maximal $O$-ideal in $C(X)$ by (i).

An ideal $M \subset C(X)$ is fixed if $\cap\{Z(f): f \in M\}$ is not empty; it is free otherwise [3, Definition 7]. According to [3, Theorem 8], the fixed maximal ideals of $C(X)$ are exactly the ideals $M(x)$. We let $\mathfrak{M}$ denote the family of all maximal ideals of $C(X)$. According to Corollary 2.4 of $[5], \mathfrak{M}$ admits the dual Stone topology $\Delta$. We denote the resulting topological space by $\mathfrak{M}_{\Delta}$. Recall that the sets $\mathfrak{D}(f)$ $=\{M \in \mathfrak{M}: f \in M\}$, as $f$ ranges over $C(X)$, form a base for $\Delta$.

Henceforth we shall assume that $X$ is a completely regular $G_{\delta^{-}}$ space; that is, $X$ is completely regular and each point of $X$ is the zero set of a member of $C(X)$ (see [1], for example).

Theorem 2. Suppose $X$ is a completely regular $G_{-}$-space and $M$ is a maximal ideal in $C(X)$. Then the following conditions are equivalent:

(1) $M$ is a fixed ideal;

(2) $M$ contains a principal primary ideal;

(3) $L(M)$ is contained in a (proper) principal ideal;

(4) $M$ is an isolated point of $\mathfrak{M}_{\Delta}$;

(5) $M$ is the union of principal primary ideals.

Proof. We show (1) $\rightarrow(2) \rightarrow(3) \rightarrow(1)$ and $(2) \rightarrow(4) \rightarrow(5) \rightarrow(2)$.

If $M$ is fixed then there is a point $x$ such that $M=M(x)$. Now there is $f \in C(X)$ such that $Z(f)=\{x\}$. Obviously $[f]$ is contained in $M$, and $[f]$ is contained in no other fixed ideal. On the other hand, 
neither is $[f]$ contained in any free ideal since $Z(f)$ is compact [3, Theorem 37]. Hence $[f]$ is a primary ideal contained in $M$ and $(1) \rightarrow(2)$.

That $(2) \rightarrow(3)$ is a direct consequence of the above corollary.

Suppose $L(M)$ is contained in the proper ideal $[f]$, which, of course, must be a primary ideal. Now $M$ is a maximal ideal and is therefore a prime and hence a prime-like ideal. By (i) above, $L(M)$ is a maximal $O$-ideal. Thus $[f] \subset M$, since, by Theorem $1, L(M)$ is a primary ideal, and since $[f]$ is contained in some maximal ideal. The zero set of $f$ is not empty since $[f]$ is a proper ideal. Pick $x \in Z(f)$. Certainly $[f] \subset M(x)$. Since $M(x)$ is a maximal ideal and $[f]$ is a primary ideal, we have $M=M(x)$. Hence $M$ is a fixed ideal and $(3) \rightarrow(1)$.

If $M$ contains the primary ideal $[f]$ then $\{M\}=\mathscr{O}(f)$, so $M$ is an isolated point of $\mathfrak{M}_{\Delta}$ and $(2) \rightarrow(4)$.

In general, if $M^{\prime}$ is a prime ideal then $k \in M^{\prime}$ implies $|k| \in M^{\prime}$ and $|k|^{1 / 2} \in M^{\prime}$, while if $|k|$ or $|k|^{1 / 2} \in M^{\prime}$ then $k \in M^{\prime}$. If $M^{\prime}$ is a maximal ideal and $M^{\prime}$ contains a sum of non-negative functions $p$ and $q$ belonging to $C(X)$, then $p \in M^{\prime}$ and $q \in M^{\prime}[3, \S 5]$. Now to show $(4) \rightarrow(5)$, suppose $M$ is an isolated point of $\mathfrak{M}_{\Delta}$. Then for some nonempty set $H \subset C(X),\{M\}=\bigcup\{\subseteq(h): h \in H\}$. Evidently for any $h \in H,[h]$ is a primary ideal contained in $M$. Choose $h \in H$ and let $k \in M$ be arbitrary. Set $f=|k|^{1 / 2}+|h|$. According to the above observations, $f \in M$, so $[f] \subset M$. Further, $[f]$ is a primary ideal, for if $M^{\prime}$ is a maximal ideal containing $[f]$ then, as we have just seen, $M^{\prime}$ contains $|h|$ and hence $h$. Therefore $M^{\prime}=M$. Now set

$$
\begin{array}{ll}
q(x)=k(x) / f(x) & \text { if } f(x) \neq 0, \text { and } \\
q(x)=0 & \text { if } f(x)=0 .
\end{array}
$$

Then $q \in C(X)$, for $Z(k) \supset Z(f)$, and if $f(x) \neq 0$, we have

$$
|k(x)|^{1 / 2} \geqq|k(x)| / f(x) \geqq 0 .
$$

Obviously, $k=q f \in[f]$, so $M$ is the union of its principal primary ideals.

It is immediate that $(5) \rightarrow(2)$, so the proof of the theorem is complete.

REMARK 1. Theorem 2 remains valid if "principal" is replaced by "finitely generated" in any of the conditions (2), (3) or (5). For in each case, it follows that $M$ contains a finitely generated primary ideal $\left[f_{1}, f_{2}, \cdots, f_{n}\right]$. But then $\left[f_{1}^{2}+f_{2}^{2}+\cdots+f_{n}^{2}\right]$ is also a primary ideal, for if a maximal ideal contains the sum of non-negative functions $f_{i}^{2}$, then it contains each $f_{\imath}^{2}$, and hence each $f_{i}$, for $i=1,2, \cdots, n$.

Remark 2. C. W. Kohls has obtained a result which yields the statement $(1) \rightarrow(2)[6, \S 4]$. 
It is natural to consider the set $P(M)=\bigcap\{[h]:[h] \supset L(M)\}$ in more detail. In the following, we suppose $M=M\left(x_{o}\right)$.

Theorem 3. Suppose $X$ satisfies the first axiom of countability. Then $P(M)=L(M)$.

Proof. Certainly $P(M) \supset L(M)$. Suppose $f \in L(M)$. We proceed to show that there exists a function $h_{o} \in C(X)$ such that $\left[h_{o}\right] \supset L(M)$ but $f \notin\left[h_{o}\right]$. Since $L(M)=N\left(x_{o}\right)$, there is a sequence $x_{n}$ converging to $x_{0}$ such that $f\left(x_{n}\right) \neq 0$ for every $n$. Choose a non-negative $h \in C(X)$ such that $Z(h)=\left\{x_{0}\right\}$. By choosing a sub-sequence if necessary, we can require that each of the sequences $h\left(x_{n}\right)$ and $f^{2}\left(x_{n}\right)$ consists of isolated points. Hence it is easy to define a continuous function $\chi$ which maps the real numbers into themselves, whose only zero is zero and such that $\chi\left(h\left(x_{n}\right)\right)=f^{2}\left(x_{n}\right)$ for every $n$. Set $h_{o}=\chi(h)$. Evidently $h_{o} \in C(X)$, $\left[h_{o}\right] \supset L(M)$ and $f \notin\left[h_{o}\right]$.

REMARK 3. It is not difficult to see that the proof of the previous theorem is valid for the space given in Example 3 of [1], even though that space does not satisfy the first countability axiom. We are indebted to the referee for the following example which shows that that theorem is not true for arbitrary completely regular $G_{\delta}$-spaces:

Let $R$ denote the real line, and let $Z$ denote the set of integers. Let $Y$ denote the union of $R$ and a one-point subset $\{x\}$ of the closure in $\beta(R)$ of $Z$. Then $P(M(x)) \neq L(M(x))$, although $Y$ is a completely regular $G_{\delta}$-space.

For let $f$ denote the element of $C(Y)$ such that $f(y)=\sin \pi y$ for all $y \in R$, and $f(x)=0$. Suppose $h \in C(Y)$ and $Z(h)=\{x\}$. Set $g(y)$ $=(\sin \pi y) / h(y)$ if $y \in R$ and $g(x)=0$. From the fact that $x$ belongs to the closure of $Z$, it follows easily that $g \in C(Y)$. Moreover, $f=g h$. Hence $f \in P(M(x))$, but $f \notin L(M(x))$.

In [8], Pursell characterized fixed ideals in terms of bounded ideals. An ideal $M$ of $C(X)$ is bounded by an element $f$ provided $f$ has no inverse, and $H(f) \supset H(g)$ for all $g \in M$. In general $H(g)=\{h \in C(X): h \neq 0$ and $h k g=h$ for some $k \in C(X)\}$. It is easy to see that $L([g])$ $=H(g) \cup\{0\}$. Similarly, $L(M)=\bigcup\{H(g): g \in M\} \cup\{0\}$. Hence $H(f)$ $\supset H(g)$ for all $g \in M$ if and only if $L([f]) \supset L(M)$. We can now establish the following connection between our Theorem 2 and the characterization in [8].

THEOREM 4. A maximal ideal $M$ of $C(X)$ is bounded by $f$ if and only if $[f]$ is a primary ideal contained in $M$.

Proof. Suppose $M$ is bounded by $f$. According to the previous comments, $L([f]) \supset L(M)$. Hence, by Theorem $1,[f]$ is a primary 
ideal, since $[f] \supset L([f])$. Also, $f$ has no inverse, so $[f]$ is contained in some maximal ideal $M^{\prime}$. But since $[f] \supset L(M)$ and $L(M)$ is primary, $M^{\prime}=M$ and $M \supset[f]$.

Conversely, suppose that $[f]$ is a primary ideal contained in $M$. Certainly $f$ has no inverse. By the corollary, $[f] \supset L(M)$, so $L([f])$ $\supset L(M)$. Hence $H(f) \supset H(g)$ for all $g \in M$, so $M$ is bounded by $f$.

\section{REFERENCES}

1. F. W. Anderson, $A$ lattice characterization of completely regular $G_{\delta}$-spaces, Proc. Amer. Math. Soc. vol. 6 (1955) pp. 757-765.

2. L. Gillman and M. Henriksen, Concerning rings of continuous functions, Trans. Amer. Math. Soc. vol. 77 (1954) pp. 340-362.

3. E. Hewitt, Rings of real-valued continuous functions I, Trans. Amer. Math. Soc. vol. 64 (1948) pp. 45-99.

4. J. G. Horne, Jr., On the ideal structure of certain semirings and compactification of topological spaces, to appear in Trans. Amer. Math. Soc. 518.

5. - On $O_{\omega}$-ideals in $C(X)$, Proc. Amer. Math. Soc. vol. 9 (1958) pp. 511-

6. C. W. Kohls, Prime ideals in $C(X)$, to appear.

7. A. N. Milgram, Multiplicative semigroups of continuous functions, Duke Math. J. vol. 16 (1949) pp. 377-383.

8. L. E. Pursell, An algebraic characterization of fixed ideals in certain function rings, Pacific J. Math. vol. 5 (1955) pp. 963-969.

UNIVERSITY OF KENTUCKY 